Parcours

anthropologiques

Parcours anthropologiques

$11 \mid 2016$

Ethnographier l'intime, les silences et les situations de violences

\title{
Une anthropologue dans un camp de réfugiés
}

Comment faire une ethnographie dans un contexte d'imposition discursive institutionnelle?

Aurore Vermylen

\section{OpenEdition}

\section{Journals}

Édition électronique

URL : http://journals.openedition.org/pa/508

DOI : $10.4000 /$ pa.508

ISSN : 2273-0362

Éditeur

Université Lumière Lyon 2

Édition imprimée

ISBN : 1634-7706

ISSN : 1634-7706

Référence électronique

Aurore Vermylen, «Une anthropologue dans un camp de réfugiés », Parcours anthropologiques [En

ligne], 11 | 2016, mis en ligne le 20 décembre 2016, consulté le 30 avril 2019. URL : http://

journals.openedition.org/pa/508 ; DOI : 10.4000/pa.508

Parcours anthropologique 


\section{Une anthropologue dans un camp de réfugiés. Comment faire une ethnographie dans un contexte d'imposition discursive institutionnelle?}

Aurore Vermylen

F.R.S.-FNRS, LAAP, Université Catholique de Louvain, Belgique

Supériorité ? Infériorité ?

Pourquoi ne pas essayer tout simplement de trouver l'autre, de sentir l'autre, de me révéler l'autre? Ma liberté ne m'est-elle donc pas donnée pour édifier le monde du Toi ?

Frantz Fanon (1952 : 229-230)

\section{PEUT-ON PARLER DE PLONGEON ?}

Rentrer sur un terrain est un moment très particulier pour un ethnologue. $C^{\prime}$ est le moment de la rencontre avec les acteurs en présence, c'est le moment de la prise de conscience d'un nouvel espace, d'un nouvel environnement. Il semblerait que ce soit aussi le moment des choix méthodologiques.

Il y a deux ans, je me rendais pour la première fois sur mon terrain d'enquête, au Burundi, dans le camp de réfugiés congolais ${ }^{1}$ de Kinama. J'y ai depuis effectué deux séjours de recherche de deux mois. Un camp de réfugiés n'est pas seulement un lieu « au bord du monde » comme le formule Michel Agier (2002), c'est également un lieu qui connait une forte confrontation des acteurs en présence. Les acteurs institutionnels d'une part, chargés de la gestion du camp, et les réfugiés d'autre part, chargés d'y vivre protégés. L'ethnologue qui se rend sur un tel terrain doit donc être particulièrement attentif à cette triple particularité ethnographique. Il doit non seulement se plier aux contraintes administratives pour mener son enquête à bien - dans mon cas il s'agissait d'obtenir une autorisation du gouvernement burundais pour rentrer dans le camp, et il doit veiller à établir un réseau de contacts aussi bien auprès des acteurs institutionnels qu'auprès des réfugiés du camp - et ce, via des canaux différents afin de ne pas - trop - se retrouver mêlé aux tensions qui pourraient exister entre les différents groupes en présence.

${ }^{1}$ Ce sont des réfugiés qui viennent de la République Démocratique du Congo. Par facilité de lecture, et puisque c'est le mot utilisé par mes interlocuteurs, je parlerai ici de Congo et de Congolais, et non de République Démocratique du Congo. 
$C^{\prime}$ est donc ce que j'ai fait. Appliquant la règle granovetterienne de la force des liens faibles ${ }^{2}$, je me suis retrouvée un dimanche midi, sur le bord d'un terrain de foot de Bujumbura, le téléphone à l'oreille, à expliquer mon projet de recherche à Mathis ${ }^{3}$, réfugié séjournant dans le camp de Kinama. C'est grâce à cette rencontre que j'ai pu être introduite auprès des réfugiés du camp sans passer par les acteurs institutionnels. C'est grâce à cette rencontre que j'ai pu proposer une observation participante avec les réfugiés du camp.

Les acteurs institutionnels, rencontrés par ailleurs, semblaient sceptiques face à l'application d'une telle méthode de travail avec les réfugiés. En témoigne cette discussion eue avec un travailleur du Haut-Commissariat des Nation Unies pour les Réfugiés (HCR) :

« [Parlant du fait que je mange avec les réfugiés de Kinama] Claude me dit qu'il y a des critères à respecter, que les règles [ici de ne pas manger avec les réfugiés] ne sont pas seulement formelles. Il me dit que je ne dois pas $\mathrm{m}^{\prime}$ attacher ou faire en sorte qu'ils s'attachent à moi. Pourquoi me dit-il ça ? N'est-ce par le propre de toute rencontre ? Quelle éthique y a-t-il derrière cette recommandation ? (Carnet de terrain, le 20 mars 2014)

D'emblée, se posait la question de comment proposer une observation participante dans un lieu où la rencontre avec les gens se fait sous la contrainte $d^{\prime}$ un formulaire d'autorisation pour rentrer dans le camp. D'emblée, j'avais l'impression de devoir imposer une posture méthodologique forte en instaurant un lien de confiance avec les réfugiés du camp. Pourquoi Claude, travailleur du HCR, avec qui je garde de très bons contacts, considérait-il que je ne pouvais pas entretenir le même type de rapport avec Mathis, réfugié congolais qu'avec lui? L'observation participante, bien qu'elle puisse se décliner très différemment, est comme un nez au milieu d'un visage pour un ethnologue : il s'agit d'une évidence méthodologique. Pourquoi, sur le terrain d'un camp de réfugiés cette méthode devrait-elle être remise en question ? La frilosité de Claude par rapport au fait de voir naitre un lien d'attachement entre mes interlocuteurs et moi soulevait-elle réellement un problème éthique, ou était-elle révélatrice d'une forme d'appréhension des réfugiés de la part des acteurs institutionnel?

\section{QUELLE PLACE POUR L'ORGANISATION SOCIALE DANS LE CAMP DE KINAMA?}

Pour comprendre les enjeux de l'observation participante en camp de réfugiés, il faut d'abord comprendre certaines des particularités ethnographiques d'un tel lieu. Un camp est de fait un espace particulier

\footnotetext{
${ }^{2}$ C'est-à-dire les ponts entre des réseaux cohésifs et indépendants. Voir Granovetter (1973).

${ }^{3}$ Les noms de mes interlocuteurs sont changés.
} 
puisqu'il est établi en urgence pour accueillir des réfugiés qui fuient un pays en guerre - ou tout au moins une situation de crise humanitaire. Théoriquement, c'est donc un lieu de transit pour ceux-ci, les réfugiés séjournant dans le camp «le temps de». Cependant, pour la plupart des camps, cette urgence devient plus ou moins permanente et les réfugiés y séjournent pendant des mois, voire des années. Le lieu camp établi en urgence devient donc un lieu de vie pour la communauté de réfugiés accueillis, arrivés soit massivement, soit au compte-goutte en fonction de la situation dans le pays d'origine. C'est en cela qu'Agier (2002) caractérise ces lieux comme des "villes-camps »: ni tout à fait des villes, ni tout à fait des camps. Le camp de réfugiés de Kinama dans lequel l'enquête a été menée a été ouvert en $2002^{4}$. Comme ces nombreuses villes-camps à travers le monde, il s'est constitué en véritable petit village, où marchés, écoles et petites habitations se partagent l'espace public. Comme ces nombreuses villes-camps à travers le monde, les drapeaux du HCR et autres ONG y flottent à son entrée.

De fait, la caractéristique première des villes-camps est qu'elles sont gérées par des acteurs humanitaires ayant pour mandat d'offrir une assistance matérielle et une protection juridique aux réfugiés. Dans le camp de Kinama, l'UNHCR, d'une part, et l'Office Nationale de Protection des Réfugiés et Apatrides (ONPRA) du Burundi, d'autre part, mettent en place un dispositif de protection et de gestion quotidienne du camp. La sécurité est assurée par des policiers burundais, l'administration du camp est gérée par l'ONPRA, et l'UNHCR se charge, avec différentes ONG partenaires, d'assurer l'aide humanitaire: logement, distribution alimentaire ou autres besoins de première nécessité, accès à la santé, à l'éducation, etc.

Cette présence massive $d^{\prime}$ acteurs institutionnels fait donc de cet espace un espace de gouvernance ${ }^{5}$, où la dichotomie entre acteurs institutionnels et réfugiés est très clairement marquée. Les réfugiés sont, certes, impliqués dans la gestion du camp, et ce à tous les niveaux - certains d'entre eux sont chargés de veiller à la sécurité du camp, d'autres sont professeurs ou infirmiers, d'autres encore sont chargés de la distribution alimentaire. Mais cette implication des réfugiés est extrêmement hiérarchisée, et ceux-ci ont des positions d'appui au bon déroulement du camp et n'ont in fine pas de réelles responsabilités. La logique humanitaire est donc pensée en termes d'organisation logistique pour veiller au bon déroulement de la vie dans un lieu de transit presque permanant.

\footnotetext{
4 L'arrivée des réfugiés congolais au Burundi a globalement commencé avec la Première Guerre du Congo (1996).

5 Pour Fesia $(2002$ : 23,24), «Au-delà de la rhétorique officielle et provisoire de la neutralité, l'espace humanitaire apparait bien comme un espace de gouvernance. » Mais, continue-t-elle, « Les enjeux liés à l'assistance matérielle et la protection juridique des réfugiés font naitre des formes d'actions publiques aux contours flous et jamais définitifs. Mise en œuvre par une diversité d'acteurs, privés comme publics, ces actions semblent bien plus souvent relever du bricolage et de réajustement permanents que d'une logique rationnelle et calculée visant à un contrôle efficace des populations réfugiées. »
} 
Et cette dichotomie s'opère au travers de règlements d'ordre intérieur. Des règlements de type éthique pour les acteurs de l'aide humanitaire qui se voient interdits de se rendre dans les quartiers des réfugiés en dehors de leurs missions, de faire des photos dans le camp ou encore de manger avec les réfugiés - et donc d'établir des relations d'attachement comme me le faisait remarquer Claude. Un règlement de type presque législatif - voire constitutionnel ? - pour les réfugiés qui se voient octroyés des droits et des obligations en exil. Ce règlement fixe par exemple les règles d'entrée et de sortie du camp, il interdit également aux réfugiés de se constituer en groupement politique. Si l'on questionne cette interdiction des relations $\mathrm{d}^{\prime}$ attachement et de constitution du politique sous l'angle anthropologique, l'on comprendra que les réfugiés en camps peuvent se sentir dénudés d'une partie de leur autonomie et donc de leur identité. En témoigne une discussion eue avec Fanta, cette femme et mère d'une dizaine d'enfant vivant à Kinama :

«Fanta est très fâchée par cette histoire de briquettes. [Les «briquettes » ont remplacées le bois comme combustible distribué pour que les réfugiés puissent cuisiner. Elles sont composées de sciures de bois et de copeaux compressés et sont une alternative écologique au bois.] Elles font beaucoup plus de fumée que le bois, elles piquent les yeux, elles sont moins pratiques, etc ${ }^{6}$. Au moment où le HCR a annoncé qu'ils changeraient le bois pour des briquettes, les réfugiés n'ont pas été d'accord. Elle dit que l'avis des réfugiés n'a pas été pris en compte, et que même si tout le camp clamait qu'il ne voulait pas des briquettes ils l'ont quand même eu. Puis, violemment, elle me montre le bidon d'eau sur lequel est imprimé le logo du HCR et me dit : 'C'est pour ça qu'on nous prend! Des hommes sans bras, sous les deux mains protectrices du HCR ?!? Ici, on n'a plus le droit à la parole...' » (Carnet de terrain, 19 mars 2014)

De manière récurrente, mes interlocuteurs me disaient se sentir vivre comme des animaux à Kinama, comme Mathis qui m'interpellait alors qu'il m'accompagnait lors d'un de mes entretiens: "sommes-nous des animaux? J'ai l'impression qu'on nous prend pour des animaux. $»^{7}$. Agier (2002 et 2008), très justement, dénonçait le fait que les réfugiés, indésirables soient parqués au bord du monde, dans des camps où ils pourraient, certes, vivre, mais en étant invisibles et en étant exclus de toute citoyenneté. L'auteur a ainsi développé la notion d'encampement pour parler du fait que les réfugiés vivent un exil prolongé dans des camps pendant des années, faute de pouvoir

\footnotetext{
${ }^{6}$ De fait, depuis que ce sont les briquettes qui sont distribuées dans le camp et non plus le bois, les réfugiés ont commencé à acheter leur propre bois de chauffage, et les briquettes ne sont utilisées qu'en dernier recours quand la famille n'a plus les moyens de s'acheter du charbon ou du bois.

7 Pour avoir eu l'occasion de faire des terrains dans plusieurs lieux d'encampement, c'est un discours que l'on entend très souvent dans les camps de réfugiés ou autres centre d'accueil. Comme une aide alimentaire et matérielle est prévue pour eux, et comme ces espaces de transit perdurent souvent pendant des années, les réfugiés sentent qu'ils ont perdu une partie de leurs responsabilités.
} 
tr` uver une s` luti $n$ durable les c`ncernant. Le HCR prév`it en effet tr` is s`luti ns durables $\mathrm{p}^{`}$ ur les pers`nnes ayant fuis leur pays et ayant tr`uvé refuge en camp : le ret 'ur dans le pays d' rigine, l'intégrati $n$ sur place et la réinstallati $n$ dans un pays tiers. $P^{`}$ ur l'auteur, dans les faits, c'est $p `$ urtant la quatrième s`luti $n$ qui préd 'mine : celle de l'encampement semi-permanant ${ }^{8}$, celle de la ville-camp.

Mais p`ur autant, le camp ne peut se transf 'rmer en ville. Al` rs que je mangeais un s`ir avec un agent du HCR et que $1^{\prime \prime} \mathrm{n}$ discutait de la manière d`nt les latrines étaient disp`sées dans le camp, celui-ci me disait que l'architecture de Kinama était pensée p` ur qu'elle ne s`it pas tr` $\mathrm{p}$ c’nf n rtable p`ur les réfugiés. "On d’it rester dans la l'gique d'un lieu de vie temp `raire », me disait-il. $\mathrm{Ou}$, p` ur le dire autrement, `n d` it rester dans la l' gique de l'aide humanitaire. D`nc dans une l'gique d’urgence semipermanente, 'ù, chaque $\mathrm{m}$ ` is, un $\mathrm{n}^{`}$ mbre très limité de pr`duits alimentaires c`ntinuent à être distribués aux réfugiés. Et, semblerai-t-il, sur le papier c`mme dans les faits, cette l’ gique ne permettrait que très peu de prendre en c’mpte les revendicati`ns des réfugiés.

$\mathrm{P}$ ur discuter de cette idée, j'aimerais revenir sur un épis`de auquel j'ai assisté quand j'étais à Kinama, au début du m` is de mars 2014. Une matinée, une missi n du Pr` gramme Alimentaire M`ndial (PAM) est pr` grammée à Kinama p`ur étudier le système de distributi` $n$ dans le camp. Cette missi` $n$ prév`it entre autres de renc'ntrer un c`mité de femmes réfugiées $p$ 'ur qu'elles puissent d`nner leur avis sur la manière d`nt la n` urriture est distribuée dans le camp. L'une de ces femmes est justement Fanta, que je cr` ise ce matin-là.

«J'arrive dans la cellule et je v`is Fanta en pagne, maquillée et avec des beaux bij 'ux. Elle se fait belle p` ur aller à une réuni` $n$ `ù elle $\mathrm{p}$ ` urra faire part de ce qui ne va pas l' gistiquement au niveau de la distributi $n$. Avec les autres femmes, elles c`mptent parler entre autre de la fumée des briquettes, qui abîme les yeux. [...] Elles disent aussi qu'ils [les réfugiés] devraient $\mathrm{p}^{`} u v^{`}$ ir ch`isir $t$ ' us les pr`duits [alimentaires] qu'ils veulent, au lieu de dev`ir ch`isir parmi une liste restreinte. Il y a beauc` up de ch`ses à vendre au marché, d`nc ce serait faisable9.

- P`urqu i ils n`us imp`sent les pr`duits ?!', me demande-t-elle, rhét riquement.

Fanta me mime enc' re une $f^{\prime}$ is le signe du HCR en mettant ses deux mains audessus de sa tête c`mme une mais`n. » (Carnet de terrain, le 3 avril 2014)

$\mathrm{P}^{`}$ ur $\mathrm{p}$ `rter ces revendicati $n s$, Fanta s’est d`nc mise en pagne. Le pagne est un habit que $p^{`}$ rtent les femmes c`ng`laises l'rsqu'elles veulent être élégantes. Ce pagne est très imp`rtant dans la culture c`ng`laise, à tel p` int

\footnotetext{
8 Ainsi, à échelle m`ndiale, le n` mbre m`yen d'années passées dans un camp p`ur un réfugié serait de 17 ans.

${ }^{9}$ A Kinama, le système de distributi $n$ est le suivant : les réfugiés reç ivent des "c` up `ns ", l'équivalent d'une m`nnaie du camp, avec lesquels ils achètent une $f^{\prime}$ 'is par $m$ ` is les pr`duits de l'aide alimentaire (restreints à une liste de quelques dix pr`duits).
} 
qu'un mariage ne peut s'envisager si un homme n'offre pas de pagne à sa femme et à sa belle-mère. Un enterrement par exemple est une situation où la femme congolaise portera un pagne. La venue d'un évaluateur extérieur du PAM, une réunion où il sera discuté des enjeux de la distribution alimentaire est également le genre de situation où une femme congolaise se dresse de son pagne. Par respect pour son interlocuteur, et pour être présentable pour mener une négociation qui est importante pour tous les membres du camp.

En tant qu'étrangère, je n'ai pas l'autorisation de séjourner dans le camp de Kinama, qui se situe à une bonne demi-heure de route de Muyinga, la ville la plus proche où je loge. J'ai donc un arrangement avec le HCR pour être véhiculée entre la ville et le camp. Le lendemain, je suis dans la voiture avec l'un des évaluateurs du PAM qui se rend dans un autre camp pour continuer son évaluation et avec qui je discute de sa mission. Nous parlons de la manière dont les distributions alimentaires sont pensées, entre autres selon des besoins nutritifs journaliers, ce qui empêche de changer la liste peu exhaustive de type de produit distribués ${ }^{10}$. Au cours de la discussion, il me dit qu'il a rarement vu un camp où les réfugiés étaient si bien portants. Il argumente en me disant que toutes les femmes du camp qu'il a rencontré avaient de beaux habits, portaient de beaux bijoux. Lui, qui avait été en Somalie, avait constaté que là-bas, a contrario d'à Kinama, les réfugiés étaient vraiment mal nourris et affaiblis.

Il s'avère que, ce jour-là, les femmes qu'il a eues l'occasion de rencontrer n'étaient pas les seules à avoir sorti leur pagne. Un vieil homme était en effet décédé quelques jours auparavant, et c'était le jour de son enterrement. Le camp, que l'évaluateur du PAM avait eu l'occasion de visiter le temps d'une matinée, vivait tout simplement un moment de deuil. Le jour de sa venue à Kinama, j'avais effectivement assisté à la cérémonie, et je notais dans mon carnet de terrain :

«Un vieil homme est décédé avant-hier. C'est le jour de son enterrement aujourd'hui. Il y a d'abord eu une cérémonie dans sa cellule. Puis, nous partons pour l'enterrer au cimetière qui se trouve à quelques pas du camp. Les femmes ont de superbes pagnes, il y a beaucoup, beaucoup de gens, ils chantent sur le chemin. Mathis et Thomas m'expliquent qu'au début les burundais étaient vraiment surpris qu'il y ait autant de monde qui suive le cadavre pour aller l'enterrer, mais c'est dans la culture congolaise d'être beaucoup pour enterrer un mort. » (Carnet de terrain, le 3 avril 2014)

10 A noter que l'aide alimentaire dans les camps est pensée selon des critères de besoins nutritifs et non pas selon des critères de besoins sociaux. Les aliments distribués (dont la liste doit comporter une dizaine de produits différents) sont calculés selon les besoins nutritifs par personne et par jour, mais ils ne couvrent pas qualitativement et quantitativement les habitudes alimentaires et culinaires des réfugiés. Ne sont donc pas comptés les besoins de "valeur psycho-affective » et de "valeur symbolique » apportés par la nourriture que Ruasse (1989) qualifie pourtant de primordiales à l'alimentation. Cependant, cette discussion avec l'agent du PAM m'a fait comprendre que l'Organisation s'attend à ce que les réfugiés échangent la nourriture reçue pour pouvoir diversifier leur alimentation. 
Cet épisode témoigne de l'articulation des perceptions radicalement différentes de chacun des interlocuteurs. Le comité des femmes, vêtues de pagnes, s'était habillé de la sorte par respect pour son interlocuteur, pour que leurs revendications soient entendues et donc par habitude culturelle. Dans l'imaginaire de l'évaluateur du PAM, chargé de constater si les besoins nutritifs des réfugiés étaient comblés quotidiennement - et donc non pas les besoins " sociaux ", l'organisation sociale n'avait que très peu de place pour se développer dans le camp. Si les réfugiés voulaient se voir octroyer une aide alimentaire plus confortable, il ne fallait pas qu'ils se présentent avec des beaux bijoux. Ce qu'il cherchait à voir, c'étaient des réfugiés, "victimes universelles » - pour reprendre les mots de Malkki (1995), pour qui il était justifié de proposer une aide alimentaire.

Dans la littérature anthropologique sur les camps de réfugiés, de nombreux auteurs (comme Harrell-Bond (1986), Zarowsky (2004), Bakewell (2001)) ont questionné cette notion de paternalisme institutionnel de l'aide en camp qui permet, notamment, d'étiqueter les réfugiés sous le statut de victime. Fassin (2006 : 16) développait ainsi la notion de gouvernance humanitaire, «c'est-àdire l'administration des populations au nom d'un principe moral supérieur qui fait de la préservation de la vie et du soulagement de la souffrance les valeurs suprêmes de l'action ». Si ce n'était sans compter les stratégies et tactiques des réfugiés, et si l'on en suit la logique humanitaire stricto sensu, les camps des réfugiés peuvent donc être considérés comme des lieux où les institutions, par leur discours et leurs pratiques, marquent le non-droit à l'organisation sociale dans le camp.

Or, justement, pour comprendre la vie en camp, il est impératif de comprendre les différentes stratégies et tactiques des réfugiés. L'aide alimentaire, à peine distribuée, est revendue dans les marchés pour que les réfugiés puissent acheter des produits de toutes sortes. Les maisonnettes en terre, à peine construites, sont alimentées en électricité pour que les réfugiés puissent recharger leurs smartphones ou regarder la télévision. L'homme mûr, à peine décédé, est enterré dignement selon les pratiques funéraires du pays d'origine. De fait, cette même littérature anthropologique a démontré que, souvent, la catégorisation de réfugié victimaire ne représentait pas une catégorie d'identification de soi, mais qu'il s'agissait plutôt d'un objet de discours pour les pratiques institutionnelles. La lecture de l'épisode de Fanta portant des revendications devant l'évaluateur du PAM nous en apprend donc plus sur la manière dont les acteurs institutionnels appréhendent les réfugiés (et la manière dont ceux-ci veulent que les réfugiés se mettent en scène) que sur la manière dont les réfugiés s'organisent au sein du camp. Par effet de miroir, lorsque l'évaluateur du PAM pense l'Autre - ici le réfugié, il ne fait que révéler son mode d'appréhension de ces acteurs, et donc au nom de quel paradigme ses actions sont mises en œuvre. 


\section{UNE IMPOSITION DISCURSIVE INSTITUTIONNELLE}

Ces dernières décennies, la représentation du réfugié dans l'imaginaire collectif occidental a fortement évolué. Pour Vanoeteren et Gehrels (2009), si, dans la foulée de la guerre froide, le réfugié était perçu comme un héros résistant à l'oppression, depuis les années 1990 et plus largement 2000, cette vision a évolué pour arriver à une projection oscillant entre pitié et suspicion.

« Au dissident soviétique, au militant de gauche menacé par les dictatures sudaméricaines, figures entourées du halo romantique de résistant à l'oppresseur, a succédé l'image d'un réfugié dépouillé de tout, sans nom, sans opinion, chassé de chez lui par des situations d'invasions brutales, d'intolérance ethnique ou religieuse, de guerre ou d'anomie. [...] les nouveaux réfugiés seraient ceux qui en ont réchappé après avoir été, pour la plupart, pris dans la tourmente d'évènements qu'ils n'avaient pu que subir. Ils correspondaient plus que tout autre à cette catégorie qui force la pitié et la compassion dans nos représentations et pour laquelle reconnaissance et réparation nous semblent justifiées: les 'victimes'. » (Vanoeteren et Gehrels, 2009 : 494)

Pour Rousseau et Foxen, qui ont étudié les procédures d'asile au Canada, les années 1960 ont représenté un tournant pour l'immigration et dans l'imaginaire collectif sur les candidats réfugiés. Au Canada, cette période a corroboré avec une augmentation de la diversité des origines des demandeurs d'asile, et donc une augmentation de la diversité des parcours de vie de ces nouveaux arrivants. Pour elles, les demandeurs d'asile étaient dès lors porteurs d'histoires multiples et complexes, peu ou mal représenté dans les médias enclins à la sur-simplification dans la présentation des conflits internationaux.

« Durant cette période, la représentation du réfugié a progressivement évolué. De la définition de la convention de Genève qui le pose en victime ayant besoin de protection, le réfugié passe à devenir un fraudeur potentiel qui veut illégitimement profiter de l'abondance de la société hôte, puis un criminel potentiel, un agresseur ayant éventuellement commis des crimes contre l'humanité, une menace à la sécurité du pays hôte. » (Rousseau et Foxen : 2006 : 506)

Les politiques de gestion migratoire en Occident ont donc été appliquées selon cette double étiquette du réfugié victime et suspect. Pour obtenir le précieux sésame du statut de réfugié, il a été demandé aux candidats à l'asile de prouver patte blanche. En d'autres termes, ces personnes étaient d'abord suspectes de ne pas être victimes avant d'être (ou non) reconnues victimes. A partir de la fin des années 1980 au Canada (1970-1980 pour la Belgique ${ }^{11}$ ), il a

11 « Après 1974, la seule porte d'entrée pour une immigration légale est celle du « demandeur d'asile ». Suite aux soupçons de fraude, la figure du «demandeur d'asile » se mue, sous la paranoïa collective, en figure $d u$ «faux-demandeur-d'asile », et le domaine de l'immigration devient une prérogative du Ministère de l'Intérieur. Ces craintes incitent l'Etat [belge] à 
été demandé aux pers`nnes v`ulant `btenir un statut de réfugié de tém`igner de leur traumatisme (Fassin : 2014), et les agents chargés de statuer sur les demandes étaient $f^{\prime}$ 'rmés $p^{`}$ ur traquer les $p `$ tentielles fraudes dans les disc' urs des candidats à l'asile.

$T^{`}$ uj urs sel`n $R^{`}$ usseau et $F^{`}$ xen (2006), le mens` nge était simultanément une stratégie de certains réfugiés face aux barrières migrat ires mises en place dans les pays les plus dével ppés, et un maintien de ces p pulati ns de ces mêmes pays à leurs fr'ntières, puisqu'il aut’ riserait ces pays à ref uler de $n `$ mbreux réfugiés sans remettre en questi` $n$ les principes de l'asile et des acc 'rds internati naux qui les balises. L'interprétati $n$ de l'hist'ire des réfugiés en termes de c`nf rmité et de déviance s'est d`nc structurée aut ur de sav ir experts et d'une expérience qui permettent une `bjectivati` $n$ de la vérité et du mens`nge - et d`nc une `bjectivati` $n$ de la victime `u du suspect, pr` $\mathrm{p}$ 'sant implicitement que cette 'bjectivati $n$ s' it p`ssible. Elles parlent ainsi $\mathrm{du}$ "mythe du réfugié menteur»: n`n seulement l'audience serait c`nstituée de telle manière à détecter les mens`nges du réfugié (qui $n^{\prime}$ existerait pas $t^{\prime}$ uj urs), mais cette audience serait ce qui renf rcerait le mythe du Canada, terre d'asile p`ur les réfugiés victimaires, les autres n'étant pas acceptés sur le s`l puisqu'il aurait été pr`uvé 'bjectivement que les demandeurs ne seraient pas, en fait, de vrais réfugiés.

Si je parle de la manière d’nt l'Occident appréhende les demandes d'asile, c'est parce que, dans le camp de Kinama, un pr' gramme au f' ncti nnement similaire a été mis en place p`ur sélecti`nner des réfugiés au départ vers des pays tiers 'ccidentaux: le pr`gramme de réinstallati`n. Et, c`mme les p` litiques de gesti` $n$ des demande d'asile en Occident, ce pr` gramme s'inscrit à l'articulati` $n$ entre la l’gique de g`uvernance humanitaire - qui s'applique sel` $n$ l'idée de s`ulager les réfugiés de leurs s`uffrances traumatiques, et la l'gique de s`uveraineté nati nale - qui catég`rise, v`ire criminalise, l'Autre extérieur à la catég`rie nati`nale.

Qu'est-ce que la réinstallati` $n$ ? La réinstallati $n$ est un pr`gramme mis en place par le HCR dans des situati`ns d'exil pr` l`ngé, c'est-à-dire quand des réfugiés ayant fui un c'nflit ne peuvent rentrer dans leur pays $d^{\prime}$ ' rigine $p$ 'ur des rais`ns sécuritaires, et quand ils ne peuvent pas $n ` n$ plus s'installer durablement dans le pays de premier accueil. Il s'agit d’nc de l'une des s` luti ns durables pensée par l'agence et qui c’nsiste à env`yer les réfugiés dans des pays tiers, maj ritairement 'ccidentaux : aux Etats-Unis, au Canada, en Eur` pe, etc. Un pr`gramme pluriannuel de réinstallati` $\mathrm{n}$ a été mis en place au Burundi. Sur les 50.000 réfugiés c`ng` lais séj urnant dans le pays, il a été

« gérer» la questi`n de l'asile par des chiffres, des st`cks, des flux, des qu` tas al`rs que jusque-là il s'agissait d'une questi` $n$ individuelle et humanitaire c`nditi nnée par des dr`its f`ndamentaux. A la fin des années $80^{\prime}$, une série de réf rmes instaurent une "phase de recevabilité » des d’ssiers. Durant cette péri` de qui sert à lever, ‘u n`n, les s`upç`ns de fraude, les demandeurs d'asile s`nt tenus d'attendre dans [une] z` ne de transit. » (Vert`ngen : 2014) 
convenu que 20.000 d'entre eux seraient réinstallés dans des pays tiers, et qu'une bonne partie des 10.000 réfugiés du camp de Kinama y prendraient part. Plus globalement, le programme burundais de réinstallation s'insère dans un programme régional considérable qui vise à envoyer les réfugiés congolais de la sous-région ${ }^{12}$. On peut parler ici de réinstallation massive puisque normalement le processus de réinstallation ne s'applique qu'à quelques réfugiés ${ }^{13}$.

Pour accéder à la réinstallation, un réfugié - ou plutôt une famille de réfugiés puisque le programme ne sépare pas les familles - doit être arrivé au Burundi avant 2005 et doit passer une série d'étapes. L'étape la plus importante se présente sous la forme d'une série d'interviews, mises en place par l'agence des Nations Unies et par les pays tiers occidentaux. Ces entretiens successifs se ressemblent les uns les autres. Lors de ces interviews, il est demandé aux réfugiés de raconter leurs parcours de vie en répondant principalement à deux questions: "Pourquoi avez-vous fui la RDC pour arriver au Burundi ? » et «Quelles sont les raisons pour lesquelles vous ne pouvez pas rentrer en RDC?». C'est sur base de ces interviews qu'il sera décidé si les réfugiés pourront partir ou non.

Comme pour les entretiens des demandes d'asile en Occident, de peur que les réfugiés ne mentent sur leur histoire, le programme s'accompagne d'une traque massive à la fraude. Cette traque à la fraude s'opère majoritairement avec les interviews ${ }^{14}$. Le fait que plusieurs entretiens successifs soient mis en place avec les réfugiés permet, par exemple, aux agents de vérifier la fiabilité des histoires des réfugiés. En interview également, les questions des agents sont orientées pour déceler un potentiel mensonge, et les agents sont particulièrement sceptiques face aux histoires des réfugiés. Ainsi, de nombreux agents chargés de réinstallation me parlaient de leurs dossiers sur base de cette conception d'histoire vraie/mensongère : "Je suis sûr que son histoire était vraie », "Je ne pourrais pas dire s'il mentait ou s'il disait la vérité », « Là, c'est sûr qu'il me mentait ».

${ }^{12}$ A l'époque de mon deuxième terrain en décembre 2014, 50.000 réfugiés congolais de la sousrégion allaient accéder au programme de réinstallation. Depuis, ce nombre a probablement augmenté. A la même époque, en effet, on annonçait que 15.000 réfugiés congolais du Burundi allaient pouvoir partir, alors que ce nombre a depuis été revu à la hausse pour envoyer quelques 20.000 réfugiés. On peut donc penser que le nombre de réfugiés congolais de la sousrégion à pouvoir bénéficier du programme a, lui aussi, augmenté.

13 Cependant, le programme tend à devenir un outil de gestion des politiques migratoires à l'échelle mondiale. Il est de fait de plus en plus utilisé par les pays tiers occidentaux, et plus particulièrement par les Etats-Unis. Notons ici que lors de la crise migratoire de l'été 2015, le programme a fait l'objet d'un accord entre les Etats membres qui tentaient de «trouver des solutions aux pressions migratoires", alors que ceux-ci peinaient à s'accorder sur une politique commune de gestion des arrivées des réfugiés. Il a été conclu que l'Europe accueillerait quelques 20.000 réfugiés «ayant besoin manifestement d'une protection internationale » par le biais de ce programme.

${ }^{14}$ Mais il est aussi demandé aux réfugiés de se dénoncer les uns les autres s'ils sont témoins de fraude à la réinstallation. Une boite anonyme est prévue dans le camp à cet effet. 
Le camp de réfugiés de Kinama est donc un lieu de forte imposition discursive institutionnelle. L'épisode des femmes réfugiées en pagne et parées de beaux bijoux nous a déjà montré partiellement la manière dont les acteurs humanitaires voulaient que les réfugiés se mettent en scène. Le programme de réinstallation et ses interviews, sésames pour accéder à un Occident tant rêvé, se met en place autour du mythe de l'objectivation de la vérité et du mensonge : on demande aux réfugiés de se raconter de telle façon à ce qu'ils prouvent qu'ils soient bien des victimes de traumatismes et donc légitimes à la réinstallation. En d'autres termes, ils doivent se raconter et raconter le Congo $\mathrm{d}$ 'une certaine manière, dans un contexte de grande méfiance institutionnelle.

Un autre lieu où les réfugiés se racontent, ce sont les ligala. Les ligala se forment lorsque quelques hommes se rassemblent pour s'échanger des nouvelles, c'est un lieu de circulation de la parole. Tout y passe : les nouvelles liées à l'organisation interne $\mathrm{du}$ camp, les discussions politiques, les naissances et les mariages, le sport, la pluie et le beau temps. Et, forcément, le Congo. Je me suis donc intéressée à ce qui se disait dans les ligala à propos du Congo. De fait, la littérature anthropologique des camps de réfugiés fait énormément référence à la nostalgie que peuvent éprouver les réfugiés de leur passé perdu. Dans son livre Purity and Exile, par exemple, Malkki (1995) témoigne du fait que ses interlocuteurs, des réfugiés burundais en Tanzanie, rigidifient leurs racines profondes en faisant de la référence au passé burundais une véritable manière de s'imaginer et de s'identifier soi-même. Ainsi, constate-t-elle que cette identité nationale est décuplée avec la mise en camp et que ses interlocuteurs se considèrent comme les seuls Hutu authentiques, purs. Un discours qui semble opposé à celui qui est nécessaire pour accéder à la réinstallation et qui demande aux réfugiés de raconter pourquoi ils sont en rupture totale avec leur lieu d'origine («Pourquoi avezvous fui la RDC pour arriver au Burundi ? » et «Quelles sont les raisons pour lesquelles vous ne pouvez pas rentrer en RDC? »; et donc, entre les lignes, «Pourquoi vous ne voudriez jamais remettre les pieds dans votre pays d'origine? »).

Mathis (qui m'avait introduite dans le camp) $\mathrm{m}^{\prime}$ avait souvent dit que les réfugiés parlaient sans arrêt du Congo dans les ligala. Mais comme il s'était depuis envolé pour le Royaume-Uni, j'ai un jour demandé à Bruno qu'il m'explique ce qui était dit dans ces ligala à propos du Congo. Il m'expliquait alors :

"Comme nous venons de différentes provinces, chacun parle des bonnes choses qui se trouvent selon la province de laquelle il vient. Ça peut être sur le plan social, sur le plan administratif, etc. Ils arrivent même à parler sur les minerais dans les sous-sols, sur la nature, etc. Et chacun va défendre que sa province est la meilleure. [...] Par exemple, 'Chez nous, la tomate prenait la couleur rouge. Et vous pouvez même semer quelques tomates seulement, vous trouverez plein de tomates. Il y a un bon fertile dans le sol. Manioc, même chose.' Mais les habitants du Katanga, ils vont répliquer 'Chez nous, il y avait les herbes que les vaches broutaient. Après la 
consommation de ces herbes, quand vous alliez traire la vache, deux vaches peuvent remplir un récipient de 10 ou 15 litres !'

Voilà ce qu'on essaye d'échanger. Et on entre en profondeur à propos de ça. Et on dit, dans notre milieu, il y avait un bon climat, qui n'était pas chaud, qui n'était pas froid. Mais ici, il y a à chaque fois la pluie, les gens glissent, les gens tombent. [...] On parle comme ça. [...] On parle souvent du Congo. C'est comme ça, on parle le plus sur notre pays d'origine. [...]

Dans notre pays d'origine, on peut trouver beaucoup de choses. Rien qu'on peut trouver ailleurs qu'on ne trouve dans notre pays. S'il n'y avait pas les troubles, s'il $\mathrm{y}$ avait la paix, on ne pouvait pas manquer de rien là-bas. [...]

On parle aussi de l'insécurité. Au Congo, vous pouvez faire une association ou de l'élevage, vous êtes en train de faire un plan de tout ça, et l'insécurité éclate. Souvent, les conflits, la guerre. Ils amènent l'insécurité que l'on parle. [...] Vous ne rentrez pas au Congo. Même les enfants que vous mettez au monde, vous leur expliquez tout ce qui s'est passé, est-ce qu'ils vont comprendre ? C'est une chaine sans fin. Est-ce qu'ils vont comprendre? Les biens pillés? Les personnes tuées? Est-ce qu'ils vont comprendre ? Avec tout ça, c'est un grand conflit qui prendra peut-être fin. [...] On parle, nous on dit qu'un jour on rentrera. Même si ça prend 100 ans ou 200 années, même si ce ne sera pas nous, ce qu'on mettra au monde, ils seront là-bas. Parce qu'ils viennent de là. » (Entretien Bruno, le 9 décembre 2014)

L'insécurité n'est donc pas absente du discours des réfugiés. Mais la volonté de retourner au Congo et la nostalgie du lieu non plus. Or, ces deux derniers aspects étaient très souvent tus par les réfugiés. A Kinama, lorsque je rencontrais sur mon chemin des personnes avec qui je n'étais pas en contact régulier, ils me décrivaient de long en large leurs parcours de vie traumatiques, date de fuite et d'entrée dans le camp à l'appui. Mais, si je leur posais des questions sur leur volonté de rentrer au Congo, sur ce qui leur manquait là-bas (même de manière détournée), les réactions étaient toujours les mêmes: Non, le Congo ne nous manque pas! Ces étonnants discours de mise en scène, lâchés à n'importe quel Blanc qui voudrait bien les entendre, étaient probablement une manière pour eux de se préparer aux interviews de réinstallation.

L'épisode des femmes en pagne portant des revendications nous l'a montré: si les réfugiés ne se mettent pas en scène comme il leur est (implicitement ou non) demandé, c'est eux qui en seront desservis. Et ils l'ont bien compris: pour s'adresser aux acteurs institutionnels, mieux vaut se raconter comme un réfugié victimaire nécessitant une protection. Mieux vaut maîtriser ces discours de mise en scène. Le programme de réinstallation accentue de fait cette imposition discursive institutionnelle, puisqu'il s'agit cette fois d'être capable de se raconter pour pouvoir accéder à un Occident rêvé. Et l'anthropologue, lui, au milieu de tout ça, doit mener son étude ethnographique. 
QUEL «MONDE DU TOI ? »

On rentre $t ` u j$ urs sur un terrain avec la v'l' nté de travailler sur une thématique particulière, ' $n$ en ress ` $r t t^{`}$ uj urs en se rendant $c^{`} \mathrm{mpte} \mathrm{qu}^{\prime}{ }^{\prime} \mathrm{n}$ a travaillé sur une autre. Le camp de Kinama n'est pas le premier dans lequel $j^{\prime}$ 'ai eu l' ccasi $n$ de mener une 'bservati' $n$ participante. En 2010, j’ai en effet passé un m` ment dans le camp de réfugiés birmans de Mae La en Thaïlande. J'étais al 'rs accueillie par une famille Karen, avec laquelle je passais mes nuits et mes j’urnées, p`ur laquelle je me prêtais à l'exercice de vendre certains pr`duits de leur petit magasin, et qui m'appelais teacher en référence au c`urs $d^{\prime}$ 'anglais que je d'nnais dans un m`nastère du camp. De ce terrain, j'avais c`nstruit une réflexi` $n$ liée à la mém `ire c`llective et individuelle des exilés : les s`uvenirs n`stalgiques et traumatiques de la Birmanie, la c`nstructi` $n$ d'une identité birmane aut’ ur de la figure d'Aung San Suu Kyi (al’rs enc` re assignée à résidence), la $n `$ ti $n$ du chez s`i.

C'est d`nc armée d'une v`l`nté de c`ntinuer cette anthr`p`l`gie de la mém `ire que je me suis rendue à Kinama. Mais un c`ntexte n'en est pas un autre, et là 'ù je p’uvais d’rmir avec les réfugiés en Thaïlande (sans aut` risati` $n$, entend 'ns-n` us bien), je ne $p^{`}$ uvais pas le faire au Burundi. De fait, le camp de Kinama est assez petit, seuls quelques dix mille réfugiés y vivent. $T$ ` ut le $\mathrm{m}$ `nde se $c^{`}$ nnait, $t$ ' ut se sait. Il y a également une présence massive des ONG et autres acteurs instituti nnels chargés de la gesti $n$ du camp. En Thaïlande par c`ntre, les camps étaient hist` riquement aut c`nstruits par les réfugiés, et le g`uvernement n'aut`risait à l'ép`que au HCR que d'être un 'bservateur extérieur, ce qui me permettait de rester $\mathrm{I}$ ' in de cette l` gique instituti nnelle. Le c`ntexte sécuritaire et hygiénique, ainsi que le rapp `rt aux Blancs n'est pas $n{ }^{`} n$ plus le même en Thaïlande et au Burundi. $\mathrm{P}$ ur ce terrain-ci, je d`rmais $\mathrm{d}$ `nc dans une famille de c`mmerçants pakistanais à Muyinga, la ville la plus pr`che de Kinama (et la seule ville de la pr`vince ‘ù les Blancs s`nt sensés d’rmir - règle du Burundi). J'avais un acc`rd avec le HCR p`ur être véhiculée jusqu'au camp, situé à une demiheure de $r^{`}$ ute de la ville. Et je devais respecter la règle qui disait que $t$ ' ut étranger au camp était aut risé à y être entre $9 \mathrm{~h}$ et $17 \mathrm{~h}$. Pas de séj ur de nuit d’nc.

Arrivant au camp avec les v'itures du HCR, j'avais extrêmement peur d'être c`nf`ndue avec un acteur instituti nnel. J'ai d`nc veillé à ce que les réfugiés sachent que j'étais une anthr` $\mathrm{p}^{`} \mathrm{l}^{\prime}$ gue, et $\mathrm{n}$ ` $\mathrm{n}$ un agent du HCR. Le premier $j$ ' ur de $m$ ` $n$ terrain, j'ai demandé à Mathis qu'il m’intr` duise auprès des réfugiés du camp :

« Je passe dans t` us les quartiers du camp. Mathis me présente, il dit que je serai là pendant un $\mathrm{m}^{`}$ ment, que je vais pr`bablement faire des entretiens avec certains $d^{\prime}$ entre eux, qu'il sera m` $n$ interprète. [...] Aussi, il insiste sur le fait que je ne suis pas du HCR, que je suis une anthr` $\mathrm{p}^{`} \mathrm{l}^{\prime}$ gue et que je travaille $\mathrm{p}$ ` ur une université en Belgique. Il explique que c'est c`mme un travail de $j{ }^{\prime}$ urnaliste. Je leur dit que 
c'est une s`rte de tém `ignage et qu'ils me dir` nt ce qu'ils aur` nt envie de me dire. » (Carnet de terrain, le 19 mars 2014)

$\mathrm{T}^{`}$ uj urs est-il que, dans un premier temps, je venais et je repartais $t^{`}$ us les j’ urs avec les v'itures du HCR. J'avais à ce $\mathrm{p}^{\prime}$ int $\mathrm{l}^{\prime}$ ' bsessi $\mathrm{n}$ de ne pas être $c^{`} f^{\prime}$ ndue avec eux qu'un s`ir, réalisant que $\mathrm{m}^{`} \mathrm{n}$ carnet d'entretiens avait exactement la c`uleur bleu ciel du HCR, je m’étais dit que c'était fichu, que m`n entrée sur le terrain avait été ratée. Dépendre des v`itures du HCR p`ur repartir en fin de j` urnée me mettait aussi s`us pressi` $n$ :

«Je me rappelle av`ir été énervée de dev` ir rentrer plus tôt à cause du HCR. Le chauffeur m'a appelé en me disant que $1^{\prime}$ ' $\mathrm{n}$ part. J'arrive, ' $\mathrm{n}$ attend enc' re une demi-heure à la v`iture et $t$ ` us les réfugiés me $v^{`}$ ient avec la bagn`le du HCR. Puis il s'avère qu'il y a quelqu'un [pr`bablement un agent chargé de la réinstallati`n] qui est resté dans le camp et que j'aurais pu rester. » (Carnet de terrain, le 27 mars 2014)

Les chauffeurs du HCR ne c`mprenaient pas vraiment cette idée de $\mathrm{l}^{\prime \prime}$ bservati $\mathrm{n}$ participante. $\mathrm{P}^{`}$ ur eux, si mes entretiens étaient finis, $\mathrm{m} ` \mathrm{n}$ travail était finit; et ils ne c`mprenaient pas $\mathrm{p}^{`} \mathrm{urqu}^{`} \mathrm{i}$ je restais dans les quartiers avec les réfugiés. $P ` u r$ finir, le fait que j'avais l'impressi` $n$ de, m`i aussi, dev`ir me plier aux c`ntraintes du HCR m’a pr`bablement permis de me rappr`cher des réfugiés. J'avais un p`int c`mmun avec eux.

Le terrain avançant, cette 'bsessi` $n$ de ne pas être c'nf ndue avec les agents du HCR s'est petit à petit diss` ute (pr`bablement aussi parce que les réfugiés c`mmençaient à sav`ir qui j'étais). En apprenant à c`nnaître les lieux et les gens, j'ai aussi c`mmencé à prendre d'autres types de transp`rts p`ur rentrer à Muyinga : le bus des réfugiés qui allaient suivre des c’urs $d^{\prime}$ 'inf 'rmatique en ville, des taxis-vél's, des taxis-m`t's ' $u$ des taxis $t$ ' ut c`urs. Bref, je me mettais à utiliser les mêmes transp`rts que les réfugiés quand je $v^{`}$ ulais $p^{`} u v^{`}$ ir rester dans le camp et que les agents du HCR repartaient plus tôt que $\mathrm{m}^{\prime} \mathrm{i}$. Mais est-ce que cette tentative d'échapper à l'étiquette de l'acteur instituti`nnel n’influençait-il pas m` $n$ regard ? C'est une questi`n que je me $\mathrm{p}$ `sais quand j'étais sur le terrain :

«Quel est m`n rapp`rt avec le HCR ? Est-ce que c'est parce que je suis avec le HCR [avec qui par ailleurs j'avais aussi des c'ntacts] que j'ai surt ut beauc' up de visi`n là-dessus? Est-ce que c'est par rapp`rt au c`ntexte de réinstallati`n que je $\mathrm{n}^{\prime}$ se pas p`ser des questi`ns de mém`ire et que d`nc j’y ai m`ins accès? » (Carnet de terrain, le 25 mars 2014)

De fait, avant de rentrer sur le terrain, ' $\mathrm{n}$ m'avait parlé du pr` gramme de réinstallati $n$. Quand je disais que je venais faire une étude dans les camps de réfugiés, les acteurs du HCR ' $u$ de l'ONPRA me disaient que beauc`up de réfugiés cherchaient à en pr`fiter, qu'ils mentaient sur leurs parc 'urs de vie et que je $n^{\prime}$ btiendrais aucune inf`rmati $n$ c`rrecte. Ils étaient sceptiques face 
aux histoires des réfugiés, j'en étais devenue sceptique de pouvoir développer une anthropologie de la mémoire à Kinama.

«Le premier contact est globalement bien passé. Le seul truc, c'est que comme j'arrive avec les bagnoles du HCR, il faut absolument que j'arrive à me distinguer d'eux. D'autant plus que, pour le moment, le HCR est dans un gros programme de réinstallation (ils vont réinstaller en Europe ou en Amérique un tiers des réfugiés), et qui dit réinstallation, dit auditions, etc etc. Tu sais comment ça marche, les mecs jouent du système, certains doivent certainement changer leurs témoignages pour décrocher le sésame. Et moi j'arrive dans ce gros bazar, je vais poser le même genre de questions et j'arrive avec leurs bagnoles... ${ }^{15}$ » (Mail envoyé à un proche, mars 2014)

Dans ce lieu d'imposition discursive, je n'osais pas m'aventurer à poser quelques questions sur le parcours de vie des réfugiés. Lors de mon premier terrain à Kinama, parler du Congo avec les réfugiés était même devenu un tabou pour moi. Ce n'était que si quelqu'un venait à $\mathrm{m}^{\prime}$ en parler que j'abordais le sujet. Je tenais absolument à laisser les questions sur les parcours de vie aux agents chargés de la réinstallation, toujours dans cette obsession de ne pas être confondue avec les acteurs institutionnels. De ce terrain, j'ai donc développé une anthropologie politique de l'organisation du camp et des tensions qui pouvaient naitre suite à la mise en place du programme de réinstallation. J'ai également développé une anthropologie des imaginaires et de la manière dont les réfugiés se projetaient dans leur future vie en Occident.

C'est donc tout naturellement que, quelques mois plus tard, j'ai suivi des réfugiés réinstallés aux Etats-Unis. Le camp n'était plus autour des réfugiés, et ce que je partageais en commun avec eux, cette fois, c'était le fait d'avoir, moi aussi, connu Kinama. Le jeu était passé, ils étaient réinstallés. Et il n'était plus question de solliciter un acteur institutionnel (ou Blanc) pour lui rappeler que le passé au Congo était extrêmement violent et synonyme de souffrance. Comme le souligne Fassin, les victimes reconnues par leur souffrance sont loin de se laisser enfermer dans une assignation à cette validation. Face au programme de réinstallation, nombreux sont les réfugiés à élaborer des ruses et stratégies pour pouvoir partir, et donc influer sur le programme qui semble imposé par le haut. C'est ce que mes interlocuteurs aux Etats-Unis me racontaient. Ils me racontaient comment ils s'étaient racontés pour pouvoir partir.

Des différents parcours qui m’ont été racontés, j'ai choisi de vous en présenter un ici, probablement un peu marginal. Thomas est Banyamulenge ${ }^{16}$,

\footnotetext{
${ }^{15}$ A la relecture, je trouve que le ton est assez sec. Je tiens cependant à le laisser tel quel parce que c'est comme ça que je l'ai écrit à l'époque. Lors de mon premier terrain à Kinama, j'utilisais ce ton-là pour parler de mon terrain avec mes proches, probablement une manière de dégager un peu d'espace pour moi dans une observation participante très prenante.

${ }^{16}$ Ethnie rwandophone de l'Est congolais, que l'on appelle souvent «Tutsi congolais », et que la plupart des congolais prennent pour des Rwandais.
} 
ou tout de moins Tutsi. Il vivait à Kinama avec sa femme et ses trois enfants, nés dans le camp. Il était professeur à l'école primaire, où il allait enseigner chaque matin. Les après-midi, il m'accompagnait parfois dans mes entretiens pour $\mathrm{m}^{\prime}$ aider en tant que traducteur. Il était également très attaché à son église évangéliste, où il jouait du piano dans la chorale. Je l'ai rencontré parce qu'il était le voisin de Mathis, la personne qui m’a introduite auprès des réfugiés. Dans le camp, chaque dix maisons sont rassemblées pour former une petite cour commune, où cuisine, lessive et autres activités quotidienne d'extérieure sont pratiquées. Cette petite cour était l'un de mes lieux d'encrage dans le camp, et je voyais quotidiennement les dix familles qui y habitaient. Ce sont ces familles qui m'invitaient la plupart du temps à manger, c'est là où je me reposais entre mes entretiens, où je cousais avec les femmes, où je laissais mes affaires en sécurité. Ce sont elles qui prenaient soin de moi et qui veillaient à ce que mon observation participante et mon travail se passe bien.

Thomas avait un esprit très carré, et était un excellent traducteur. Là où d'autres réfugiés entraient dans de grandes conversations avec mes interlocuteurs, Thomas prenait la peine de me traduire phrase par phrase ce qui était dit, savait où arrêter le discours de l'autre pour prendre le temps de me traduire l'histoire racontée. Il aimait prendre son temps pour m'expliquer comment le camp fonctionnait dans ses moindres détails. Je lui donnais mon appareil photo et il allait faire un tour du camp pour me ramener quelques clichés. C'est donc un réfugié avec qui j'appréciais travailler lorsque j'avais besoin de faire un mapping du camp ou une étude sur le fonctionnement des institutions. Il réfléchissait aussi beaucoup à qui je pourrais rencontrer dans le camp, il veillait à ce que j'ai un contact avec chaque organisation du camp. Il voulait également que je le suive certains dimanches à la messe dans son église évangéliste. Une tradition familiale personnelle ne me donnait pourtant pas envie de le suivre dans cette aventure, et j'ai laissé l'église au croyant. Par contre, je l'ai suivi lorsqu'il me proposait de rencontrer le pasteur, ou d'autres réfugiés, la plupart appartenant à son ethnie. De fait, il était membre actif et fier de la communauté des banyamulenge et ne cachait pas qu'il ne voulait en aucun cas être confondu avec une autre ethnie, qu'il ne voulait pas que ses enfants jouent trop avec des enfants de Bafuliro, ethnie avec laquelle les Banyamulenge sont en conflit.

Le programme de réinstallation étant mis en place, il me racontait aussi les différentes stratégies qu'il avait mises en place pour pouvoir partir aux EtatsUnis. Il me chuchotait que son père et son frère étaient dans le camp, mais qu'ils prétendaient appartenir à des familles différentes pour pouvoir se voir être réinstallés plus rapidement. Les trop gros dossiers étaient examinés plus tard que les petits, me disait-il. Il me racontait aussi comment il était arrivé seul dans le camp, puis comment il avait été cherché une femme dans son village d'origine pour pouvoir fonder une famille. Et puis, cheminant sur quelques sentiers du camp, il me racontait une anecdote qu'il avait vécue à Kigali, au Rwanda. Je demandais ce qu'il avait fait au Rwanda. Silence. Il 
restait évasif et me disait « $\mathrm{T}$ ` $\mathrm{i}$, tu aimes $\mathrm{p}$ `ser beauc` up de questi ns. ». Un autre $\mathrm{j}$ ur, revenant sur les détails de ce qui lui était demandé de dire en interview, il me disait : "Ils demandent si `n a déjà t’ uché des armes. Tu te rends c`mpte? B`n...».

L`rs de m`n sec`nd séj ur à Kinama en n`vembre et décembre 2014, Mathis s'était env` lé $\mathrm{p}^{`}$ ur le R`yaume-Uni, `ù il avait été réinstallé. Th mas me demandait si j'avais des $n^{\prime}$ uvelles de lui. Mathis ne lui d'nnait plus aucunes n`uvelles depuis qu'il était parti, et Th`mas en épr`uvait un sentiment empreint de c`lère et de tristesse. Il se rappelait avec $\mathrm{m}$ ` $\mathrm{i}$ des m`ments que $n^{`}$ us avi`ns passés avec Mathis dans le camp, était tr` $p$ fier $p^{\prime}$ ur lui env`yer de ses n` uvelles, mais me demandait de le faire $p^{`}$ ur lui. Il disait que, lui, quand il serait réinstallé, il ne ferait jamais le silence, c`mme $\mathrm{d}^{\prime}$ autres réfugiés le faisaient une $\mathrm{f}^{\prime}$ is partis. Th mas était un réfugié qui cachait aux autres réfugiés ses stratégies p` ur partir aux États-Unis.

Ce pr`gramme de réinstallati $n$ avait un én `rme $p$ `ids dans le qu` tidien des réfugiés, qui, p`ur la plupart, rêvaient de partir dans les pays tiers ‘ccidentaux. $\mathrm{P}$ ur Th`mas, partir aux Etats-Unis représentait le rêve de sa vie. «Tu te rends c`mpte? L'indice téléph 'nique des USA c'est le 001. C'est le premier pays au m`nde! Je vais dans le pays 'Number one' ! " Le pr' cessus était cependant très l’ng, et il p`uvait se passer plusieurs années entre la première interview et le départ effectif. J'avais l'impressi' $n$ que Th 'mas tr` uvait en ma présence une échappat’ire $\mathrm{p}^{\prime} \mathrm{ur}$ me rac`nter $t$ ' utes les ch`ses qu'il ne v`ulait/p` uvait pas dire aux autres. Dans le camp, les réfugiés, prêts au départ, se regardent de fait s` uvent en chien de faïence face au pr`cessus de la réinstallati` $n$.

L`rs de m`n sec`nd terrain à Kinama, Th`mas passait les dernières interviews avec les Etats-Unis, et une semaine ' $u$ deux après que je $s$ ' is rentrée en Belgique, il apprenait qu'il avait été sélecti`nné p`ur le départ. Un hasard de calendrier fait que je me suis rendue à Bukavu du 15 au 26 avril 2015. M`n v`l de ret`ur était planifié depuis Bujumbura. Arrivé à l’aér` p`rt, surprise! Je v`yais Th`mas et sa famille, ainsi que six autres familles de réfugiés, qui partaient $p^{`}$ ur les États-Unis, en passant par Bruxelles. On était d`nc dans le même $v^{`} l$, et j’ai d`nc pu suivre ses impressi`ns $l^{`}$ rs de ce m`ment crucial.

Je suis partie aux États-Unis en 'ct’ bre 2015, p`ur dix j’urs. Th 'mas travaillait à la chaine $\mathrm{p}$ ur une entreprise qui faisait des burgers. Il était resp 'nsable de mettre les `ign`ns surgelés dans les burgers. Les h`raires étaient harassants et ce travail le fatiguait beauc' up. Heureusement, il avait le dimanche p`ur lui. Il avait tr`uvé une petite église évangéliste dans le c`in et s'était intégré à la c`mmunauté. Il n’avait pas n`n plus `ublié ses pr` p`s virulents envers les ethnies autres que la sienne. Al`rs qu'il ne c`nnaissait que très peu de c`ng lais ( $v^{\prime}$ ire très peu de gens t’ut c`urt), il me demandait p` urqu` i je passais du temps avec des Bafulir` . "Tu sais, ces gens ne s`nt pas fréquentables ». Il tenait le même genre de pr`p`s avec les réfugiés irakiens, 
réinstallés eux aussi dans la même rue que lui (cette rue ressemblait en effet à Resettlement village, et des réfugiés du monde entier y habitaient : congolais, burundais, somaliens, irakiens, birmans, etc.).

Un soir, Thomas semblait nostalgique de l'Afrique. De Kinama d'abord, où il me reparlait de différentes personnes que j'avais rencontrées. De l'époque avant Kinama ensuite. Son entourage et sa position sociale lui manquait surtout. Alors que nous rentrions à l'appartement, discutant toujours de ces souvenirs, il me dit: «Tu vois, ce qui me manque le plus c'est de tenir une arme ». Nous étions dans ce petit escalier sombre et étroit qui montait vers l'appartement. Il s'était arrêté et s'était mis à chuchoté. « Tu sais, ma femme ne va pas aimer que je te raconte ça. » Mais la confidence s'était déclenchée. Il voulait me dire ce qu'il avait caché aux agents de réinstallation.

Qu'avait-il caché ? Qu'il était un enfant soldat d'abord. Recruté enfant pour quelque milice tutsi de la région des Grands Lacs. De cette époque, ce qui lui manquait le plus c'était d'arriver dans les villages avec les miliciens, et de pouvoir ainsi terroriser tout le monde. "Les gens avaient peur de moi, Aurore !», me disait-il. L'enfant soldat avait grandi, les tensions politiques de la région avaient changées. Il était maintenant recruté par un seigneur de guerre, tutsi également, et avait monté en grade. Il était maintenant chargé de recruter des enfants qui pourraient aller se battre pour des milices. Au fil des années, ses missions avaient évoluées. Sa dernière mission en date avait été de se rendre dans le camp de Kinama et d'y trouver des jeunes tutsis qui pourraient partir au front à l'Est du Congo. "J'en ai recruté 24 ! », me disait-il fièrement et fiévreusement. C'était une partie de lui qu'il m'avait forcément caché quand il était à Kinama. Le fait de ne plus y être lui permettait de me raconter des choses qui appartenaient désormais à un passé. Il n'avait plus personne à qui cacher ce secret. Plus personne n'était là pour valider qu'il était un «bon réfugié », professeur de français dans l'école primaire du camp. L'imposition discursive n'était plus.

Cette histoire aurait hérissé les poils des agents de réinstallation, qui disaient connaitre et tolérer les stratégies et mensonges des réfugiés pour se voir être réinstallés. Ces agents me disaient que les personnes qu'ils cherchaient vraiment à arrêter, c'étaient les criminels de guerre. Que c'étaient eux qui représentaient un réel danger pour la sécurité des pays tiers. Un d'entre eux me disait ainsi ne pas jouer le jeu humanitaire, qu'il «ne portait pas les lunettes roses du HCR », mais qu'il était sûr qu'il y avait des " criminel de guerre qui passent $»$.

Dans Peau noire, masque blancs, Fanon concluait par ces lignes: "Supériorité ? Infériorité ? Pourquoi ne pas essayer tout simplement de trouver l'autre, de sentir l'autre, de me révéler l'autre ? Ma liberté ne m'estelle donc pas donnée pour édifier le monde du Toi ? » (Fanon : 1952). L'aide humanitaire en camp, ainsi que les politiques de sélection des réfugiés statutaires semblent avoir choisi leur «Monde du Toi ». J'en choisi un autre. C'est-à-dire que je choisi un «Monde du Toi » non-empreint du jeu de la 
catégorisation des politiques migratoires qui tournent autour de la figure de l'Autre objectivement suspect ou victime. Mais je reconnais que c'est probablement une posture que je prends a posteriori, après mon expérience de terrain à Kinama et aux Etats-Unis. La volonté de m'extraire de la posture d'agent institutionnel a probablement, dans un premier temps, joué un rôle inverse sur mon travail. Certes, j'avais réussi à faire comprendre aux réfugiés que j'étais un autre type d'acteur, mais j'étais moi-même sceptique des histoires des réfugiés. De fait, je ne posais pas de questions sur les parcours de vie des réfugiés, pour ne pas être confondue avec les agents, mais aussi par peur que les réfugiés me racontent des histoires de mise en scène. L'imposition discursive institutionnelle accouche de fait de sollicitations massives de la part des réfugiés qui, à tout bout de champ, se racontent comme ils devraient se raconter en interview. Dans ce brouhaha d'histoires, il est facile pour l'ethnologue de devenir sourd. Et la mémoire a cette étrange manière de sélectionner les informations retenues selon les cadres de pensé délimités a priori. Je crois donc que ce que je cherchais à voir, c'étaient ces histoires stratégiques des réfugiés, et ce n'est probablement pas par hasard que je vous présente celle de Thomas.

A posteriori, je suis malgré tout contente de n'avoir posé aucune question aux réfugiés quant à leur parcours de vie. Je crois que sur le terrain de Kinama, qui connait un programme de réinstallation qui demande aux réfugiés d'expliquer pourquoi ils sont partis du Congo, je n'aurais pas pu faire des entretiens semi-directifs avec ce type de questions. Mais ça m'a aussi permis de me rendre compte que les parcours de vie que reçoivent les anthropologues étaient reçus au compte-goutte, et qu'ils étaient certainement complexes. Thomas, certes, a fait partie des milices de la région. Mais il a également d'autres facettes que j'ai également eu l'occasion de découvrir. Et toutes celles-là ensemble me permettent également de comprendre des enjeux de la sous-région, de comprendre partiellement qui sont ces personnes qui font partie de cette main d'œuvre de la guerre, disponible dans les Grands Lacs. Le décentrement ethnographique proposé par l'observation participante permet de fait à l'anthropologue de regarder ses interlocuteurs avec des lunettes non plus roses - pour reprendre les termes de l'agent chargé de réinstallation en parlant de la politique humanitaire du HCR, non plus noires - celles de la suspicion, mais kaléidoscopiques - celles de la complexité contextuelle.

\section{BIBLIOGRAPHIE}

Michel AGIER, Au bord du monde, les réfugiés, Paris, Flammarion, 2002.

Michel AGIER, «Between war and city : Towards an urban anthropology of refugee camps ", Ethnography, 3, 3, 2002, pp. 317-341 
Michel AGIER, Gérer les indésirables. Des camps de réfugiés au gouvernement humanitaire. Paris, Flammarion, 2008.

Olivier BAKEWELL, « Uncovering local perspectives on humanitarian assistance and its outcomes », Disasters, 24 (2), 2000, pp. 103-116.

Frantz FANON, Peau noire, masques blancs, Paris, Seuil, 1952.

Didier FASSIN, «L'humanitaire contre l'Etat, tout contre», Vacarme, Paris, association vacarme, 2006, pp. 15-19.

Didier FASSIN, «De l'invention du traumatisme à la reconnaissance des victimes. Genèse et transformation d'une condition morale. ", Vingtième siècle. Revue d'histoire, Paris, Presses de Sciences Po, 2014, pp. 161-171.

Marion FRESIA, «Aide humanitaire et production de services publics en Afrique de l'Ouest: le cas de la gestion des populations mauritaniennes réfugiées au Sénégal ", Bulletin de l'APAD, vol. 23-24, 2002 [En ligne]. URL : http:/ / apad.revues.org/146

Mark GRANOVETTER, "The strength of the Weak Ties", American Journal of Sociology, vol. 78, n 6, Chicago, University of Chicago Press, 1973, pp. 1360-1380.

Barbara HARREL-BOND, Imposing Aid: Emargency Assistance to Refugees, Oxford, OUP, 1986.

Liisa MALKKI, Purity and Exile: violence, memory, and national cosmology among Hutu refugees in Tanzania, Chicago \& London, The University of Chicago, 1995.

Cécile ROUSSEAU et Patricia FOXEN, « Le mythe du réfugié menteur : un mensonge indispensable, ", L'Evolution Psychiatrique, vol. 71, n³, 2006, pp. 505-520.

Alain VANOETEREN et Lys GEHRELS, «La prise en considération de la santé mentale dans la procédure d'asile », Etude. Revue du droit des étrangers, $\mathrm{n}^{\circ} 155$, numéro spécial, 2009, pp. 492-543.

Youri-Lou VERTONGEN, " De quoi 'sans-papiers' est-il le nom ? », Revue Nouvelle, $\mathrm{n}^{\circ}$ 6-7, juin-juillet 2014, [En ligne]. URL : http:/ / migrationsetluttessociales.blogspot.be/2015/06/de-quoi-sans-papiers-est-ille-nom.html

Christina ZAROWSKY, “Writing Trauma : Emotion, Ethnography, and the Politics of Suffering Among Somali Returnees in Ethiopia", Culture, Medecine and Psychiatry, 28, 2, 2004, pp. 189-209. 
RÉSUMÉ: Comment édifier un «Monde du Toi » dans un camp de réfugiés? Cet article propose de penser comment faire une ethnographie face aux politiques de gestion des réfugiés basées sur un imaginaire victimaire ou suspicieux de la figure du réfugié. Face à cette imposition discursive institutionnelle, qui demande aux réfugiés de se mettre en scène ou de se raconter selon leurs parcours de vie traumatiques, comment faire anthropologie ? Cet article se base sur un travail de terrain dans le camp de réfugiés congolais de Kinama au Burundi, où un programme de réinstallation a été déployé. La réinstallation vise à envoyer les réfugiés des camps dans des pays tiers occidentaux de manière définitive.

MOTS-CLÉS : Imposition discursive institutionnelle, camp de réfugiés, réinstallation, ethnographie. 